tent," are alike in principle; but by neither of us, I believe, was this principle first invented. For last summer I saw, in a drawer in the small room adjoining Sir J. Simpson's ward in the Edinburgh Infirmary, a spring tent similar to the three just mentioned. I could learn nothing about it, except that it had been there a long time: Perhaps these few lines may draw the attention of your readers to the subject, and someone may be able to give us the history of this discarded but interesting little instrument.

The instrument-maker tells me that he made my first "spring tent" early in 1864.

I am, Sir, your obedient servant,

Sheffield, March 20th, 1866.

J. H. Aveling, M.D.

\section{THE PHYSIOLOGY OF THE LUNGS.}

To the Editor of THE LArceT.

SIR,--It is far from my wish to raise any captious objections to the conclusions which any physiological writer may put forth as the result of his observations; and I would much prefer cordially to agree and corroborate him where I could conscientiously do so; and it is a matter of sincere regret that I feel it incumbent on me now to enter a protest against some statements made by Dr. Waters in a paper read before the Medico-Chirurgical Society on Dec. 12th, 1865, and published in The LanceT on Dec. 23rd, and to which my attention has only just been drawn.

Dr. Waters states that " the true respiratory portion of the lungs consists of a series of air-sacs, situated at the extremity of each bronchial tube." This, to begin with, is far from a correct statement. If it were true, each of the small bodies which Dr. Waters calls "air-sacs," and which I have called "leaflets," would be detached from the remainder of the lung, and, as a consequence, there would be no intercommunication of air between adjoining or adjacent "leaflets" or air-sacs. That there is a very free intercommunication may easily be shown. If quicksilver be made to flow by means of a capillary tube, such as is used for the injection of the lymphatics, through a minute bronchial tube into a leaflet, the quicksilver, having filled the cavities of the leaflet, will flow backwards through numerous bronchial tubes in the vicinity, and if these be closed by liga. ture then the quicksilver will spread itself into other leaflets and return by more distant bronchial tubes. If any of the leaflets be examined carefully it will be found that not one minute bronchial tube alone terminates in it, but that numerous bronchial tubes in all directions around the base of the leaflet enter it, establishing a very complete anastomosis with the other similar tubes by their all communicating with the same air-cells in the cavity of the leaflet in which they terminate.

If these leaflets be further examined it will be found that, though they are each so separated by means of fissures as to be almost distinct bodies, yet that, in point of fact, none of these are wholly detached from those which adjoin them, but they are continuous with other similar bodies in some part of their circumference, and that the continuity of each one of these bodies with those which surround them is not in any case wholly severed; thence it occurs that the vascular plexus formed by the final distribution of the pulmonary artery on the walls of those bodies constitutes one continuous network of anastomosis throughout the whole lobule, and in a modified form throughout the whole lobe.

The sulci formed between these leaflets leave the channels through which the radicles, constituting the commencement of the pulmonary veins, dip down to reach the trunks of the veins which run along the under surface of each of the larger bronchial tubes.

Dr. Waters goes on to say that "the pulmonary arteries are the only bloodvessels distributed to the "air-sacs." " He adds, "They are engaged not simply in carrying blood for the special function of the lungs, but also for the nourishment of the tissue to which they are distributed. Although the bronchial arteries pass along the bronchial tubes and the areolar tissue of the lungs, they send no branches to the walls of the airsacs, which are solely occupied by the plexus formed by the pulmonary artery. In speaking of the areolar tissue of the lungs the author wished it most clearly to be understood that no such tissue is found in the walls of the air-sacs." To every one of these statements I am compelled to give an explicit contradiction, which I wish to do with every respect and courtesy to Dr. Waters. These are matters which admit of actual demonstration, and are questions of fact which can be brought to the test of the eyesight. I am ready to produce any number of unequivocal specimens where every part of the tissue of the lungs, including the leaflets, is abundantly supplied with capillary bloodvessels injected from the aorta, through the bronchicl (so-called) arteries; these specimens will prove that these capillary bloodvessels are distinct in all their characteristics, and totally unlike those of the pulmonary vessels.

When I wrote my treatise on this subject I was of opinion that it was impossible to inject the pleura from the so-called bronchial artery; I have since, however, succeeded in injecting the pleura of an inflamed human lung by causing the fluid to flow through the so-called bronchial artery from the aorta. These facts prove that the tissue of the lungs is wholly supported by means of the so-called bronchial artery, and not at all by the pulmonary, the distribution of whose capillaries is altogether unsuited for any such purpose. Dr. Waters, however, is right when he asserts that the first damage accruing to the lungs in cases of red hepatization is through the pulmonary vessels. Any cause which produces an arrest, greater or less, of the whole circulation will manifest its action in the first instance by the retardation of the blood in the pulmonary vessels, producing a mechanical congestion in the plexus of the bronchial membrane (which exclusively belongs to the pulmonary system) and in the true pulmonary plexus surrounding the leaflets. This is synonymous with the impairment to that extent of the vital force which ought at that time to be engendered by the respiratory combinations; and, in a slight form, is the true patloology of an ordinary attack of "cold" with a certain amount of bronchial or pulmonary congestion, and is usually remedied by restoring the eirculation to its original state but carried to a greater length, or protracted so as to allow a transudation of the blood into the cavities of the leaflets, the condition called "red hepatization" ensues. This result can easily he imitated in injecting the pulmonary vessels. But the structure of the lungs, or a portion of them, being thus damaged by the causes producing red hepatization, in which the pulmonary bloodvessels have been concerned in a simply mechanical manner, if death does not immediately ensue, fresh action is set up, and the proper vessels for the nourishment of the tissue of the lungs-viz., the so-called bronchial arteries-are immediately brought into play, and, like all other vessels in the body similarly circumstanced, become enlarged in proportion to the increased function which they are now called upon to discharge: having to remedy, or strive to remedy, the injury which the transuded blood thus occasions to the tissue of the Iungs. It is true that the energy with which these bronchial arteries will set about their task will be in proportion to the degree in which the pulmonary vessels in undamaged portions of the lungs will discharge their function of combining with the air, and thus supply the bronchial (so-called) vessels occupied in repairing the damaged portion with the vital condition requisite for that purpose; but the pulmonary vessels did not produce the damage in the first instance, except in the mechanical way above described, and they take no direct part in the restorative action, which endeavours to weave together the damaged tissue by the appropriate bloodvessels. This function is wholly discharged by the bronchial bloodressels, whose physiological attributes are precisely opposite to those of the pulmonary capillaries.

It is quite evident that nothing appertaining to the tissue of the lungs can influence the greater or less quantity of blood which passes through the pulmonary vessels. This is wholly governed by the greater or less quantity of blood which the greater or less activity of function in the body at large may cause to flow towards the lungs, and the greater or less demand for arterial blood which the functions of the body at large may occasion, together with the vital orgasm which the combinations in the lungs must at the same time supply to the whole body in order to enable it to employ the arterial blood and to create the venous blood which is to fill the pulmonary capillaries.

Nothing in the whole range of physiological and anatomical science can be more plain, more thoroughly established by evidence, and more susceptible of ocular demonstration, than that the so-called bronchial vessels are distributed to every part of the lungs, the tissue of the leaflets included, that they discharge all the duties which relate to the maintenance of the tissue of the lungs, and that they have no anastomosis whatever with the pulmonary vessels, and are not mingled with them in function in any way.

With respect to the last part of the quotation which I have made from Dr. Waters' paper-namely, that the areolar tissue of the lungs is not found in the walls of the air-sacs (leaflets), I must contend that here also Dr. Waters is quite in error. 
When the pulmonary vessels are perfectly injected, if they be immediately inspected, all the capillary bloodvessels will then be visible and distinct, because the so-called "areolar" tissue is then transparent; but if the specimen be left in fiuid for some time, the interstitial tissue swells up, becomes semiopaque and flocculent, and it will be found a tedious and long business, occupying many days, before all this loose tissue can be pulled away by the forceps, so as to leave the bloodvessels again distinctly visible. In thus pulling the tissue away it is dragged from the sulci which separate the leaflets, and then the form and shape of the leaflets become distinct.

If, instead of adopting this plan, strong spirit of wine be used for the preservation of the specimen, the flocculent interstitial tissue will shrivel up and become adherent to the fibrous tissue of the leaflets, and a person examining such a specimen might easily imagine that no interstitial tissue was present. But a specimen in strong spirit, as above described, could not be preserved as a slide for the microscope. When such a specimen is put into more dilute spirit the interstitial tissue soon becomes apparent, because it then becomes flocculent and opaque. I can exhibit many specimens in which this interstitial tissue has been wholly removed, others in which it has been partially removed, and others again in which it is not at all removed.

In the discussion which ensued on the reading of the above paper, a remark was made respecting the exudation of the contents of the pulmonary vessels into the interlobular tissue. I may observe that from the peculiar distribution of the pulmonary vessels such an event is almost impossible. In injecting those vessels extravasation may easily be made into the interior of the leaflets, producing a condition resembling red hepatization, and a frothy exudation from the plexus in the bronchial mucous membrane analogous to the peculiar expectoration of pneumonia is also easily produced, but never the result which resembles hrmoptysis, nor that state of infiltration of the interlobular tissue which goes by the name of " pulmonary apoplexy ;" but both of these states are very readily made manifest by an extravasation while injecting the so-called bronchial vessels.

I am, Sir, your obedient servant,

Winchester, Feb. 28th, 1866. JaMes NEwTON HEALE, M.D.

\section{THE ACTUAL CAUTERY FOR NÆVI.} To the Editor of THE LANCET.

SIR, - A brief notice of lessened pain caused by the hot iron in the destruction of nævi may induce your provincial readers more frequently to use it in place of nitric acid and caustics, and in cases where the ligature is inadmissible, or fails to arrest the vascular growth. I feel assured it is the least painful of all modes of treatment when freely and properly applied. The child ceases crying a few moments after oil is used; whereas after nitric acid it cries for hours, if much has been used to abrade the surface, the reapplication of which fails to destroy large diffused nævi. The double ligature passed through the base of the nævus and tied on either side, where the position of the growth does not forbid its use, or where skin is not implicated so as to distort the features, is perhaps the best, though a painful, mode of treatment.

Three cases lately cauterized by me illustrate well the use of this plan. An infant three weeks old I cauterized on the upper eyelid for a circular nævus, which was growing rapidly, and would soon have produced ptosis. Two applications quite arrested its growth; on the second, the nævius was raised by a tenaculum passed through it, which was burned down upon. Months have elapsed since the operation, and the only residue is a whiter mark than the skin where the vascularity was.

Another case was that of the smallest seven months' child I ever saw reared. A nævis appeared soon after birth at the extreme edge of one nostril, which, on examination, was found to pass up the orifice for some distance. At nine months old many applications of the nitric acid had been used, giving great pain; but the growth continued to increase. I applied the actual cautery freely to the nævus, passing it up the nostril, and using some momentary tension with the semi-button-like head of the curved instrument, to press well into the depression near the point of the nose. This has totally arrested the growth, leaving no deformity (so important in such a position)

The third case I shall trouble you with was that of a child a year old, with a large raised nævus of the middle of the cheek, with large varicose-looking vessels attached to it running back to the neck; the stain apparent through the cheek on opening the mouth. The nitric acid had twenty times been applied, the child crying for hours after its use. The ligature was thought inadmissible in London from the large surface that would be implicated. I applied a cautery half the circumference of the nævus in the middle of it; it appa. rently gave little pain, which soon ceased. After the surface had healed, I found it had so contracted that the same instrument covered the remaining part, which, having very florid edges, was spreading again. The second application has arrested all morbid growth; the vascularity gone; the large vessels almost imperceptible. I attribute all this to the known contraction of the cicatrices of burns. There is no distortion of feature, but a white irregular scar. Under these circum. stances, I feel myself at liberty to urge the use of the nearly obsolete cautery in these cases. Such instruments are easily formed with the file from flat-headed bolts, nails, and screws, obtainable of every country ironmonger.

Hounslow, March, 1866. I am, Sir, yours faithfully,

\section{MODIFICATION OF THE KNEE-JOINT SPLINT USED AT KING'S COLLEGE HOSPITAL. To the Editor of THE LANCET.}

SIR,-The modification in this splint, originally made at my suggestion by Mr. Matthews, of Portugal-street, W.C., con. sists of a movable thigh-piece, acting by a slot-and-set screw;
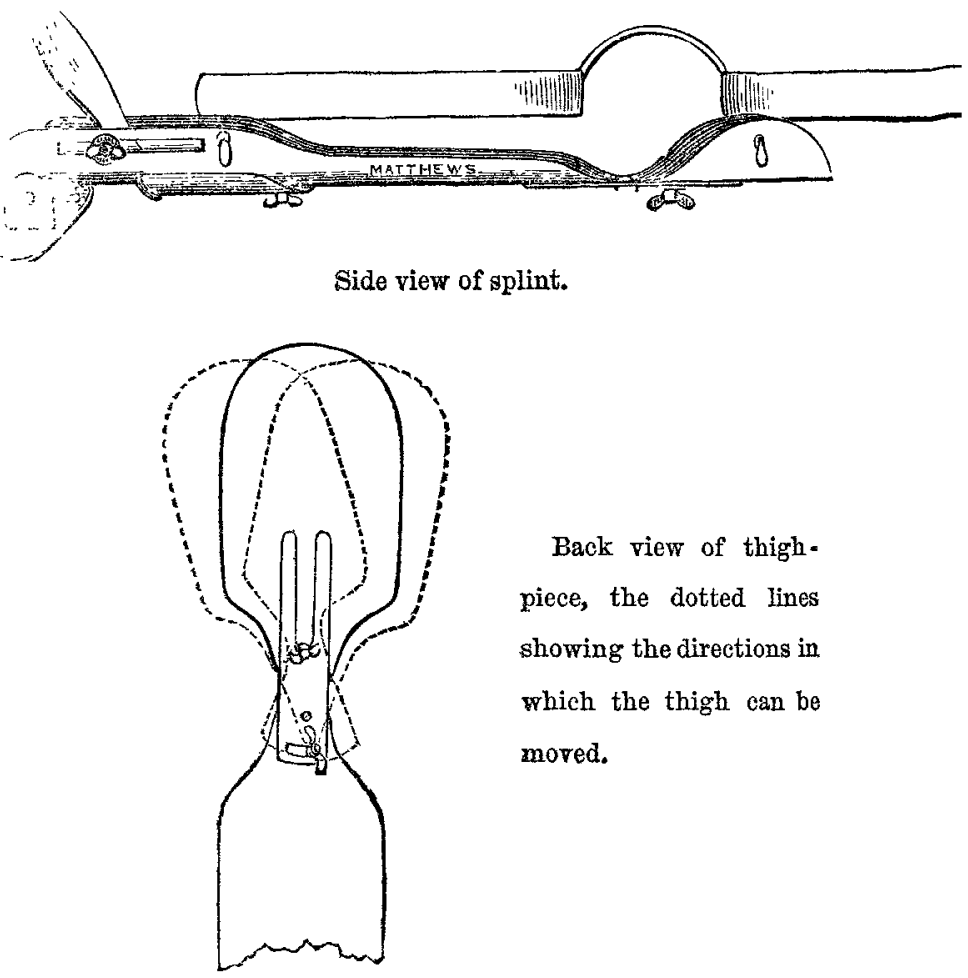

Back view of thigh. piece, the dotted lines showing the directions in which the thigh can be mored

by which arrangement any tendency of the joint to grow out wards or inwards after excision of the knee can be rectified, instead of using pads on either side-a somewhat troublesome and painful proceeding formerly in use. The splint has been used in several cases, and found to answer its intended purpose I am, Sir, your obedient servant, New Brighton, Birkenhead, March, 1866. Wrlugam Beli.

\section{NEW VACOINATION BILL. To the Editor of THE LANCET.}

SIR,--Through the medium of your journal we beg to call the attention of the Poor-law medical officers and public vaccinators of the metropolitan district to a meeting held by the medical officers of the Fulham Union on March 13th, to consider the proposed Vaccination Bill. At this meeting it was proposed-

"That a fee of $2 s .6 d$. and $3 s$. $6 d$. should be substituted for 1s. $6 d$. and $23,6 d$, in consequence of the responsibility in. volved and the trouble necessitated by the several certificates required by the proposed Bill."

"That in successful revaccination the same fee should be paid, inasmuch as it will involve more trouble than primary 